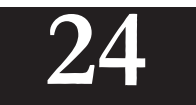

\title{
EvoluCión DE LA
} INDUSTRIA

\section{AUTOMotriz eN MÉXICO}

en el siglo XX y la primera década del siglo XXI

Juan José Huerta Mata

Ruth Maria Zubillaga Alva

Universidad de Guadalajara

Recibido: 28 de abril de 2015

Aceptado: 10 de octubre de 2015

\begin{abstract}
RESUMEN
I 1 sector automotriz es 4 un motor de desarrollo Ude la economía nacional que al día de hoy representa un importante porcentaje de la Inversión Extranjera Directa (IED). México ha fabricado motores a nivel industrial desde el año de 1925, algunos años después de que esta industria iniciara formalmente en Europa. La experiencia alcanzada por los productos automotrices hechos en México a lo largo de casi noventa años, ha posicionado al país dentro de los principales productores

a nivel mundial. La industria automotriz formada por el sector terminal y de autopartes se localiza en nuestro país en las regiones económicas Centro, Norte, y Centro Occidente, principalmente. Esta última, se encamina a ser la segunda en importancia en el país con respecto a esta industria, por lo que es sustancial realizar acciones y proyectos de carácter local enfocados a fortalecer la cadena productiva con un consecuente desarrollo del mercado interno regional y beneficios a niveles micro y macroeconómico.
\end{abstract}


Palabras clave: Desarrollo económico, Industria automotriz, Regiones geográficas

\section{Abstract}

The Automotive Sector is an engine for the national economic development and today it is an important average in foreign direct investment. Mexico has making engine to industrial level in 1925 years ago, some years late the automotive industry start in Europe. The experience achievement for the automotive products made in Mexico along ninety years, have positioning the country indoor the mains producers the level world. The Automotive Industry is forming for the terminal sector and auto parts are localized mainly in economics regions, Center, North, and Occident Center. This last, walk to be the second in importance in the Country in this sector; for the principal is do actions and projects of local type, focus to grounded the productive chain with you development in local and regional market and benefits to micro and macroeconomics levels.
Key words: Economic Development, Automotive Industry, Geographics Zones

\section{INTRODUCCIÓN}

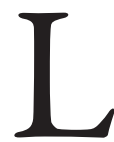

a Industria Automotriz en México tuvo su inicio cuando Buick instaló oficialmente la primera planta armadora de autos en nuestro país. Ford siguió sus pasos en 1925, y en los siguientes años se incluyeron otras firmas.

El objetivo principal en este artículo es analizar la situación actual de la industria automotriz, particularmente de la Región Centro Occidente de México; el objetivo específico es determinar las condiciones actuales de operación de las plantas automotrices y sus relaciones con empresas de otros tamaños, instituciones académicas, organismos de gobierno y otros.

Durante el mandato del presidente Díaz Ordaz, en los años sesenta, y con la intención de impulsar el desarrollo tecnológico y la creación de empleos, se emitió un decreto que dictaba que las plantas automotrices establecidas en México deberían ensamblar todos los automóviles comercializados en el país, regulando también el porcentaje de autopartes importadas. Algunas empresas estuvieron en desacuerdo con esta disposición, y abandonaron el país, sin embargo, otras continuaron y están presentes al día de hoy.

Esta industria se ha consolidado afrontando, a lo largo de los años, los retos que impone la variable económica tanto en nuestro país como a nivel mundial, al grado que actualmente México es el séptimo productor mundial de automóviles (INA, 2014).

México posee una posición geográfica privilegiada, siendo el país más septentrional de América Latina y frontera con Estados Unidos; además, costos de operación muy competitivos, experiencia probada en la fabricación de vehículos y autopartes de clase mundial. Estos y otros factores hacen de nuestro país una excelente opción para atraer la inversión en este rubro. Particularmente, la región Centro Occidente de México, alberga cinco empresas ensambladoras de autos: General Motors, Honda, Mazda, Volkswagen y Nissan, y dos armadoras de vehículos pesa- 
dos: Cummings e Hino. Estas plantas se concentran en los estados de Guanajuato, San Luis Potosí, Jalisco y Aguascalientes (Miranda, 2007).

\section{Antecedentes GENERALES}

$\mathrm{E}$ l "viejo mundo" fue la cuna de la Revolución Industrial, periodo de la historia que se caracterizó por la gran cantidad de invenciones tecnológicas y científicas que cambiaron para siempre el modo de producción tradicional artesanal, y con ello, todo el sistema social y económico. Una de las más importantes innovaciones de esta etapa fue la máquina de vapor, ampliamente utilizada para mover mecanismos y aparatos diversos como bombas, motores marinos, locomotoras, y, por supuesto, los primeros automóviles. A continuación se mencionan algunos de los antecedentes más importantes.

Nicolás-Joseph Cognot, inventor francés, en 1769 construyó un automóvil de vapor al que llamó El Fardier, y posteriormente otras dos versiones, de las cuales la tercera se encuentra expuesta en el Museo Nacional de la Técnica en París.
Partiendo de esta base, durante los años siguientes se sucedieron numerosos inventos con innovaciones como el freno de mano, velocidades, volante y motor de combustión interna, por mencionar algunas; $y$ movidos posteriormente con aceite, células eléctricas, gas de carbón y, finalmente, gasolina. En 1903 se puso en circulación en Londres el primer taxi del mundo, el cual funcionaba con nafta.

En 1897 se fundó la "Societe des automóbiles Peugeot", en Francia, que utilizaba motores Daimier para sus modelos. Las primeras compañías automotrices en ese país fueron Panhart et Levassor y Peugeot, iniciando con esto la producción masiva de automóviles.

André Citroën fue una de las grandes figuras del siglo $\mathrm{XX}$ en el mundo empresarial; inició en 1919 y en un lapso de diez años ya estaba construyendo uno de los imperios industriales más importantes. Con una visión innovadora logró llevar a su empresa al éxito, aunque ello no lo salvó de enfrentar la crisis económica de 1934 y la posterior quiebra.

En la actualidad, esta marca aún está vigente en Europa, junto con la mundialmente famosa Volkswagen, que tuvo sus inicios en la Alemania nazi, cuando Hitler tuvo la idea de que se construyera un auto para el pueblo, para que todos los alemanes pudieran tenerlo (Agüero, 2014).

El 8 de marzo de 1934 se expone el modelo de auto popular sistema "Porsche". El 22 de junio del mismo año se celebra el contrato de la Industria Automotriz del Reich con la sociedad Porsche para la construcción del primer prototipo Volkswagen, que significa en alemán "auto del pueblo". Louis Renault, que construyó su primer automóvil en 1899, llevó a su empresa a ser una de las más importantes de la industria automotriz, pero luego de la II Guerra Mundial fue acusado de colaborar con los alemanes y enviado a prisión. Murió en 1944 y la fábrica pasó a manos del estado francés, con el nombre de "Regie Nationale des Usines Renault", nombre que conserva al día de hoy. En 1899 August Horch fundó la empresa A. Horch \& Cie en Alemania; al perder un litigio sobre la razón social, cambió a la traducción latina AUDI de 
su apellido (en español significa "escucha").

En 1932 se unieron Audi, DKW. Horch y Wanderer, para formar Auto Union AG. Los 4 anillos de su emblema representan a cada una de las marcas alemanas que la formaron. La producción de esta empresa incluía motocicletas y autos de carreras de alta tecnología. Durante la Segunda Guerra Mundial solo produjo vehículos militares, en la posguerra fue expropiada y desmantelada por la ocupación soviética. Resurgió en 1949 con el nombre de Auto Union $\mathrm{GmbH}$, que en 1958 se integra a Mercedez Benz, y en 1964 Volkswagen adquiere la mayoría de las acciones de Auto Union GmbH. Después se fusionó con NSU Motorenwerken AG, en 1969; ya en 1985 cambió su nombre por Audi AG, y a partir de entonces la empresa (especializada en autos de lujo) y sus productos llevan el mismo nombre (Autopasión s.f.).

La famosa fábrica de automóviles de Baviera BMW (Bayerishe Motoren Werke), fundada en 1913 originalmente para construir motores para la aviación, cuyo logotipo de la marca simboliza una hélice con el azul del cielo y a su vez representa los colores de la bandera bávara. Después de la Primera Guerra Mundial empezaron a construir motocicletas, y en 1929, su primer automóvil.

Mientras tanto, en el nuevo continente, Henry Ford, ícono del automóvil en América, funda su compañía en 1903, y en 1908 presenta su modelo " $T$ ", un auto más accesible, resistente y al alcance de las masas. Su éxito se logra al crear el sistema de cadena de montaje, además de la intercambiabilidad de sus piezas; cada parte del auto se fabricaba con tolerancias estrictas, de modo que cada modelo era igual a cualquier otro, lo que permitía que se pudiera producir en grandes cantidades y a precios más bajos, mientras que otras compañías producían sus automóviles de manera laboriosa, artesanal. En 1909, debido a la gran demanda del modelo T, inauguró una fábrica de gran magnitud en Michigan (donde introdujo el sistema de cadena de montaje), dando inicio así a la moderna industria del automóvil. Al cabo de algunos años la producción se perfeccionó y los precios descendieron. En un año de producción en Esta- dos Unidos circulaban 10000 modelos "T", y cuando dejó de producirse, en 1927, se habían vendido más de quince millones en todo el mundo. Fue la primera empresa en utilizar la cadena de montaje y con algunas prácticas empresariales importantes para la época: no se trabajaba más de 8 horas diarias, se procuraba la rotación de personal para evitar el tedio, sus trabajadores eran mejor remunerados que otras organizaciones, entre otros incentivos.

Otra empresa automotriz estadounidense de relevancia fue General Motors Corporation, que se funda en 1908 por William Durant, con sede en Michigan, Estados Unidos, incorporando a Buick Motors Co., y posteriormente Oldsmobile. Sus principales marcas son Chevrolet, GMC, Buick y Cadillac; en México se hace presente en 1935.

Otra de las marcas de renombre de los Estados Unidos de Norteamérica la crea Walter Chrysler, ex trabajador de la Buick Motor Co., quien lanzó su primer auto, el Chrysler Six, en 1924. En 1934 y aconsejado por Orville Wright, construyó un túnel de viento para realizar pruebas sobre los contornos de 
las carrocerías con el propósito de desarrollar el sistema airflow, aunque en la actualidad pertenece al grupo Daimler Benz adquirida en 1998 (Historia Chrysler, 2013).

Destacan en el sector automotriz latinoamericano Brasil y México, dos grandes potencias latinoamericanas en producción y venta de automóviles. Estos países se destacan desde la llegada de Volkswagen a territorio brasileño en la década de 1950 y al mexicano en la década de 1960, época en la que el modelo "Escarabajo", el VW sedán, se convirtió en un auténtico símbolo en ambos países. En la actualidad Brasil y México concentran el 90\% de la producción de la región; el ranking de mayores empresas del sector automotriz en Latinoamérica en 2009 según ventas, lo encabezan 5 empresas brasileñas y 5 mexicanas (de empresas matrices europeas, norteamericanas y japonesas como Volkswagen, Fiat, General Motors y Nissan) (Rico, 2015).

La producción automotriz en Asia se inició más tarde, prácticamente solo se puede mencionar a Japón con esta industria en el mismo periodo de tiempo que América. En 1902 se creó un vehículo de baja potencia (doce caballos de fuerza) y otros modelos producidos de manera artesanal, que de ninguna manera competían con los autos importados, principalmente Ford y General Motors, que ya se comercializaban en la isla. Incluso estas compañías instalaron ensambladoras en el país nipón, aunque con partes importadas. La producción japonesa se centró antes y durante la guerra en fabricar camiones militares. Las primeras compañías automotrices autorizadas, Nissan, Toyota e Isuzu, iniciaron operaciones con grandes ventajas en la legislación tributaria, aunque con una producción de apenas 800 autos por mes, en contraposición con las restricciones aplicadas a Ford y General Motors, que terminaron saliendo del país en 1939.

Al terminar la Segunda Guerra Mundial, y debido a la ocupación estadounidense que sufrió Japón, este país eliminó su gasto militar, introdujo reformas económicas que le permitieron dedicar sus recursos a impulsar su economía. Se dio nuevamente prioridad a la industria del acero, que desde su inicio fue manejada por el gobierno. La cooperación entre empresas y el sector gubernamental logró una rápida recuperación en la posguerra, lo que se llamó el milagro japonés (Aquino, 2000).

Kiichiro Toyoda produce en 1937 el primer prototipo de automóvil y establece las bases para la compañía Toyota Motor Company Ltd. Al finalizar la Segunda Guerra Mundial, la empresa japonesa Toyota ya era el mayor fabricante de vehículos de Japón, con más del 40\% del mercado. Implantó el Sistema de Producción Toyota o Lean Production, (producción ajustada), en contraposición con el sistema fordista de producción en cadena. El sistema de producción toyota requiere equipos de trabajadores calificados y maquinaria versátil para producir componentes variados de acuerdo a la demanda de los consumidores. Implica un sistema flexible, y entregas just in time o justo a tiempo y la producción ajustada que evita costos de almacenamiento. El sistema de calidad implantado por esta empresa con criterios como cero errores, orden y limpieza del área de trabajo o 5's, mejora continua, etcétera. 
Toyota es al 2009 el primer fabricante de automóviles más importante del mundo, con 7'813,000 vehículos producidos; en ventas de unidades y también en ventas netas (Álvarez, 2010).

Además de la eficiente estrategia gubernamental, incidieron otros factores externos en el auge de esta industria, como la demanda de camiones requerida por Estados Unidos en la Guerra de Corea (1950), el libre comercio de autos comerciales y automóviles (1965); y la unión de Japón a la Organización para la Cooperación y el Desarrollo Económico (OCDE), facilitando acuerdos y uniones con empresas automotrices de países desarrollados.

En 1972 se promulgó la Ley de Control de la Contaminación, después de la cual las empresas japonesas Honda, Suzuki y Toyo Kgyo (hoy Mazda) realizaron importantes aportaciones otorgando a Japón uno de los estándares más estrictos en control de contaminación del mundo. En 1973 la Organización de Países Exportadores de Petróleo (OPEC por sus siglas en inglés), incrementa considerablemente los precios del petróleo, con la consecuente demanda de los pequeños y económicos autos japoneses.

En la década de los 80 la alta demanda de los autos japoneses ya se resiente en Estados Unidos, que solicita acuerdos para equilibrar la balanza comercial. En esa etapa la demanda era tal que, por ejemplo, en México había lista de espera para adquirir un automóvil Tsuru. A partir de 1990 se presenta una desaceleración de la economía japonesa, que afecta a las diez empresas automotrices debido a que la capacidad instalada supera la demanda.

Por otra parte, en 1960 Corea del Sur ensambló autos para el mercado local, y al igual que en Japón, la participación del gobierno fue decisiva en la prosperidad de la industria del automóvil. A partir de 1970 se dio énfasis en la producción de autos a gran escala mediante el "Plan de Promoción a Largo Plazo de la Industria Automotriz" lanzado en 1974 (Jenkins, 1995). Hyunday Motor Company, entonces ensambladora de Ford, lanzó su modelo "Pony", el cual tuvo gran aceptación que le sirvió para obtener aún más apoyos del Estado consistentes en créditos blan- dos, subsidios y condiciones preferenciales (Cuéllar, 2012).

En estos países la intervención del Estado resultó trascendental al enfocarse en la vinculación de la educación con las exigencias de las industrias terminales y demandando a estos programas de aprendizaje obligatorios. A partir de 1976, Corea del sur comenzó a exportar autos y en la actualidad es uno de los principales exportadores en el mundo.

En China en 1950 la FAW (First Automobile Works Group Company), que hoy tiene como socios a Mazda, Toyota y Volkswagen, inició producción bajo el auspicio de la ex Unión Soviética. En la década de los cincuenta y sesenta estuvo prohibido el comercio con Japón, y el hermético sistema político limitó el intercambio tecnológico y comercial ocasionando el subdesarrollo de esta industria y de la economía china en general. A partir de 1978 con la Reforma Económica China, la apertura y estrategias colocaron a este país como uno de los más atractivos para inversión extranjera. Consiguieron pasar de un país tercermundista a ser la segunda economía mundial, después de 
Estados Unidos. Habría que decir también que en esta economía la producción ha estado orientada básicamente a la maquila y el ensamble, y aunque la tecnología china tiene aún que mejorar para alcanzar un nivel competitivo, ya emprendió este camino en 2004 exportando modelos propios producidos por la FAW. Así pues, la economía china antes estancada y empobrecida es actualmente líder (Cuéllar, 2014).

En India, Suzuki está presente desde 1983 y es la empresa automotriz número uno, aunque también hay plantas armadoras de GM, VW, Honda, Ford y Nissan. Además de ser el sexto exportador mundial de automóviles, es relevante la demanda local, por ser una economía de clase media emergente, además de las altas tarifas aplicadas a los vehículos importados. Para satisfacer el mercado interno se producen automóviles de bajo costo. La empresa hindú Tata Motors construye el Nano, auto más barato del mundo (Cuéllar, 2014).

\section{LA INDUSTRIA AUTOMOTRIZ EN MÉXICO}

$\mathrm{L}$ as empresas fabricantes de automóviles establecieron plantas en México debido a los bajos costos de operación además de que era más barato importar los juegos para ensamble que los autos terminados, aunque la infraestructura era pobre y la productividad baja al menos abastecía el mercado interno.

En el periodo de 1940 a 1970, dentro de lo que se le llamó "El milagro mexicano", se dio un crecimiento económico sostenido que transformó al país en una nación moderna e industrializada. En el sexenio de Adolfo López Mateos ya se hablaba del propósito de integrar la industria automotriz en México, lo que se puso en marcha en 1962 durante el mandato de Díaz Ordaz. Se emitió un decreto automotriz, el de integración nacional, el cual prohibió la importación de automóviles y camiones; estas regulaciones dictaban que las empresas establecidas en México debían ensamblar todos los automóviles comercializados en el país, prohibiendo la importación de motores y equipos para ensamble, y regulando también el porcentaje de las autopartes importadas a un
40\% máximo. El propósito de esta medida fue desarrollar una industria automotriz nacional, promover la creación de empleos además de fomentar el progreso tecnológico. Algunas firmas decidieron salir del país por incompatibilidad o incapacidad de cubrir el requisito, entre ellas las europeas $\mathrm{Ci}$ tröen, Fiat, Volvo y Mercedes Benz, quedándose en México Chrysler, Chevrolet, Datsun (hoy Nissan), Ford, General Motors, Renault y Volkswagen (Vicencio, 2007).

La política económica del país se basó en la sustitución de importaciones, o modelo ISI, Industrialización por Sustitución de Importaciones (Vidal y Guillén, 2007), que es una estrategia que se siguió en México como en otros países latinoamericanos para producir internamente los insumos que se importaban de los países desarrollados, esto a raíz de la escasez de partes ocasionada por la Segunda Guerra Mundial y de la Gran Depresión. El gobierno federal impulsó el surgimiento de empresas de autopartes al emitir dos decretos más, el de desarrollo automotriz, que determinaba que el $60 \%$ del capital social 
debería ser nacional; y el decreto de 1977 para el fomento de la industria automotriz, que favorecía a las empresas terminales mediante la facilitación de divisas con la finalidad de integrar a los productores nacionales a las cadenas productivas de las grandes empresas ensambladoras. El modelo ISI fue acertado para ese tiempo, sin embargo, esta política proteccionista al interior, aunque consolidó la industria automotriz, provocó que las empresas no tuvieran competitividad hacia los mercados extranjeros, y que al paso de los años, dio lugar a elevados niveles de Inflación, devaluación de la moneda (1976), deuda externa y altas tasas de interés con la consecuente fuga de capitales, situación que detonó en la crisis de 1982 durante el gobierno de José López Portillo.

A partir de la década de los setenta el sistema político ya empezaba a ser un poco más flexible con las importaciones, aunque tratando de mantener los precios bajo control y el intercambio de divisas balanceado, permitiendo la entrada paulatina de vehículos, ya que la producción en el país resultaba insuficiente a la deman- da de unidades. Los decretos publicados en los años 1978 y 1989 liberaron el manejo de divisas y permitieron la importación de nuevos modelos, y la firma del Acuerdo General de Aranceles y Tarifas (GATT) en 1987; lo anterior facilitó las condiciones hacia el Tratado de Libre Comercio de América del Norte (TLCAN), como medidas para enfrentar la crisis económica de los años 19821987 (Álvarez, 2002).

Durante el sexenio de Carlos Salinas de Gortari se amplió el límite de partes importadas, al establecer el límite de contenido nacional a un $36 \%$ (desde 1962 era de 60\%), situación que obligó a la industria de autopartes a ser más competitiva, aunque todavía estaba prohibida la importación de autos compactos. Finalmente, se firmó el TLCAN en 1994 entre Canadá, Estados Unidos y México, cambiando el modelo económico a Modelo Multinacional, con una desregulación paulatina para el sector automotriz. A partir de 2004 en la importación de automóviles nuevos y autopartes provenientes de Estados Unidos y Canadá los aranceles quedaron totalmente elimina- $\operatorname{dos}^{1}$. Desde ese año, cualquier persona podría importar vehículos nuevos de estos países y las empresas amadoras ya no están obligadas a cumplir con la balanza comercial positiva (que el valor de exportaciones fuera mayor que el de importaciones), que estuvo disminuyendo gradualmente desde la firma del TLCAN.

Gracias al libre comercio, las plantas automotrices antes ubicadas en el centro del país, Ford y Chrysler, comenzaron a desplazarse a otras regiones más específicamente a la frontera norte, por su cercanía con las empresas automotrices localizadas en Estados Unidos y para abastecer el mercado externo, y posteriormente se desplazaron a la región centrooccidente. Para integrarse a la cadena de producción las empresas de autopartes instaladas en la Ciudad de México y Querétaro también se mudaron a la frontera norte y a la región centro-occidente integrándose en agrupamientos productivos llamados clusters.

\footnotetext{
1 Se refiere a la regla de origen que obliga a los plantas armadoras a incorporar un porcentaje de proveedores de insumos nacionales o regionales a la cadena productiva, a pesar de utilizar en su mayoría materias primas de equipo original extranjera.
} 
En el año 2000 se celebró el tratado de libre comercio con la Comunidad Económica Europea, que fomentó el diálogo político y la cooperación con la Unión Europea. En 2003 se publicó el decreto para el apoyo de la competitividad de la industria terminal y el impulso al desarrollo del mercado interno de automóviles. Actualmente México cuenta con doce tratados y acuerdos comerciales con 44 países. El sistema legal y político del país favorece el desarrollo de nuevos negocios y ofrece seguridad jurídica para la inversión (ProMéxico, 2013).

La clasificación arancelaria de los productos que ingresen al país se rige por la Ley de los Impuestos Generales de Importación y Exportación, que en la regla octava complementaria del Artículo Segundo, se concede tratamiento preferencial para la importación a las plantas armadoras, previa autorización de la Secretaría de Economía (ProMéxico, 2013).

Actualmente a la industria automotriz son aplicables algunos instrumentos de apoyo como los Programas de Promoción Sectorial (Pro- sec, 2002)2 cuya creación, de acuerdo con la Secretaria de Economía, "obedece a la necesidad de elevar la competitividad del aparato productivo nacional en los mercados internacionales, de promover la apertura y la competitividad comercial con el exterior como elementos fundamentales de una economía dinámica y progresista, así como de establecer instrumentos para fomentar la integración de cadenas productivas eficientes".

Adicionalmente, el TLCAN establece que a partir del octavo año de su vigencia la modificación de los mecanismos de importación temporal vigentes en los países miembros, con el fin de evitar la distorsión de las preferencias arancelarias acordadas en el marco del mencionado tratado, y que, con tal propósito a partir del 1 de enero de 2001 se igualó el tratamiento arancelario que México otorga a insumos y maquinarias no norteamericanas empleados para la producción de mercancías destinadas a los tres países que integran el mercado nortea-

2 Decreto por el que se establecen diversos programas de promoción sectorial, Diario Oficial de la Federación, 2 de agosto de 2002. mericano. Además, que la proveeduría no norteamericana de insumos y maquinarias es crítica para ciertas industrias y que estas requieren contar con condiciones arancelarias competitivas para abastecerse de insumos y maquinarias no norteamericanas. En atención a lo anterior, el gobierno federal en México decidió establecer condiciones competitivas de abasto de insumos y maquinaria para la industria exportadora y propiciar una mayor integración nacional de insumos (Secretaría de Economía, 2013).

\section{LA INDUSTRIA} AUTOMOTRIZ, ASPECTOS GENERA-

\section{LES}

e puede entender como
industria automotriz "al
conjunto de empresas que conforman la industria terminal y la industria de autopartes; conociendo a la empresa de la industria terminal como una empresa constituida u organizada de conformidad con la legislación mexicana, que está registrada ante la Secretaría de Economía del gobierno federal y que se dedica en México a la producción o ensamble final de los vehículos automotores; 
por otro lado, en cuanto a la industria de autopartes, son empresas que operan y producen autopartes constituidas u organizadas de conformidad con la legislación mexicana" (Decreto para el Fomento y la Modernización de la Industria Automotriz, AMIA, 31 de mayo de 1995).

$\mathrm{La}$ industria automotriz, como la rama de la manufactura que se encarga del diseño, desarrollo, fabricación, ensamblaje, comercialización y venta de automóviles, se integra de un sector terminal constituido por plantas ensambladoras de automóviles, y de un sector de autopartes integrado por empresas productoras de partes y componentes para equipo original y de repuesto. Esta industria es una gran generadora de empleo, ya que además de la mano de obra directa que requiere, influye en toda una industria paralela de autopartes, además de la consecuente mano de obra indirecta de las empresas vinculadas a este sector.

Sector Terminal. Este sector de la industria automotriz está conformada por plantas ensambladoras de automóviles, camiones, tractocamiones y autobuses. Es el eje de la industria automotriz al abarcar cerca del $73.1 \%$ del total, correspondiendo el $26.9 \%$ restante al sector de autopartes. Representa un indicador del desarrollo y modernización del país, ya que implica que otras ramas productivas que intervienen en la cadena de manufactura han alcanzado el nivel requerido. Ejemplos de estas ramas son la industria de textiles, aceros, plásticos y químicos, así como las redes de comercialización y logística.

En el país se encuentran instaladas plantas ensambladoras de diez de los principales fabricantes de vehículos ligeros con dieciocho complejos en once estados; y once fabricantes de vehículos pesados, concentrados en las regiones norte, centro y centro-occidente. Además de contar con más de treinta centros de diseño automotriz (figuras 1 y 2 ) (ProMéxico, Industria Terminal Automotriz, 2013).

Según la Industria Nacional de Autopartes (INA) (2013), por varias décadas la producción automóviles se destinó a consumo nacional, sin embargo, a partir de la celebración del Tratado de Libre Comer- cio de América del Norte (TLCAN) el enfoque se dio hacia la exportación. Últimamente el mercado interno se ha visto beneficiado gracias a los financiamientos a mediano plazo para la compra de automóviles, además de una baja en los precios desde el año 2002.

Según información de ProMéxico (2013), en 2012 México conservó el octavo lugar mundial en producción de vehículos, con 3.02 millones de unidades; octavo lugar mundial en producción de vehículos ligeros, con 2.88 millones de unidades (12.8\% más que en 2011); séptimo lugar mundial como productor de vehículos pesados con 138,078 unidades; y cuarto lugar como exportador de estos vehículos. La manufactura de automóviles representa cerca del $73 \%$ de la producción total de la industria automotriz. Al día de hoy se fabrican más de 48 modelos de vehículos en el país.

Este sector engloba el beneficio de la generación de empleos especializados a gran escala (en 2012 se generaron 62,196 empleos, según ProMéxico), y la importante recaudación fiscal que de esta se obtiene (en 2011 el aporte al 
PIB fue del $4 \%$ y el $20 \%$ de la producción manufacturera).

En cuanto a las exportaciones, se ha registrado un decremento debido a la caída de la demanda de vehículos en Estados Unidos, que es el principal destino de exportación, a consecuencia del éxito de los autos japoneses y europeos importados o fabricados en territorio estadounidense. Esta situación es determinante también para México, ya que las principales inversiones en plantas automotrices son de las empresas estadounidenses Ford, General Motors y Chrysler (ProMéxico, Industria, Terminal Automotriz, 2013).
Figura 1. Localización de plantas ensambladoras en México de vehículos ligeros.

\begin{tabular}{|c|c|c|}
\hline Empresa & Ubicación & Productos \\
\hline Chrysler & $\begin{array}{l}\text { Ramos Arizpe, Coah. } \\
\text { Saltillo, Coah. } \\
\text { Toluca, Edo. Mex. }\end{array}$ & $\begin{array}{l}\text { Motores V8 Hemi } 5.7 \\
\text { y } 6.1 \text { lts. Camiones, } \\
\text { pick up RAM, } \\
\text { estampados } \\
\text { Journey, PT Cruisier } \\
\text { estampados, refac. }\end{array}$ \\
\hline Ford & $\begin{array}{l}\text { Hermosillo, Son. } \\
\text { Chihuahua, Chih. } \\
\text { Cuautitlán, Edo. Mex. }\end{array}$ & $\begin{array}{l}\text { Fusión, Milán, MKZ, } \\
\text { estampados } \\
\text { Motores gasolina } 4 \text { cil. } \\
\text { Fiesta }\end{array}$ \\
\hline General Motors & $\begin{array}{l}\text { Ramos Arizpe, Coah. } \\
\text { Silao, Gto. Toluca, Edo. } \\
\text { Mex. San Luis Potosí, } \\
\text { SLP }\end{array}$ & $\begin{array}{l}\text { Chevy, HHR, Captiva, } \\
\text { motores, estampados, } \\
\text { Camiones, Escalade, } \\
3500, \text { Silverado, } \\
\text { Suburban, Sienna y } \\
\text { Cheyenne. Silverado, } \\
\text { motores } 4 \text { y } 8 \text { cil. } \\
\text { fundición. Autos Aveo } \\
\text { (estampado). }\end{array}$ \\
\hline Honda & $\begin{array}{l}\text { El Salto, Jal. Celaya, } \\
\text { Gto. }\end{array}$ & $\begin{array}{l}\text { CR-W, MUV, } \\
\text { autopartes Honda y } \\
\text { Acura, Accord. }\end{array}$ \\
\hline Mazda & Salamanca, Gto. & $\begin{array}{l}\text { Motores Skyactiv de } \\
\text { Mazda } 2 \text { y } 3\end{array}$ \\
\hline Nissan & $\begin{array}{l}\text { Aguascalientes I, } \\
\text { Aguascalientes II, } \\
\text { CIVAC, Mor. Lerma, } \\
\text { Edo. Mex. }\end{array}$ & $\begin{array}{l}\text { Platina, Clio, Sentra, } \\
\text { Tiida, motores gasolina } \\
4 \text { cil. Estampado, } \\
\text { transeje, fund. Tsuru y } \\
\text { Tiida, Fundición. }\end{array}$ \\
\hline Toyota & $\begin{array}{l}\text { Tijuana, B.C. } \\
\text { Salamanca, Gto. }\end{array}$ & Pick up, Tacoma y cajas \\
\hline Volkswagen & Puebla, Pue. Silao, Gto. & $\begin{array}{l}\text { Beetle, Bora, Jetta, } \\
\text { SportW y motores }\end{array}$ \\
\hline
\end{tabular}




\section{5 novage

\begin{tabular}{|l|l|l|}
\hline AUDI & San J. Chiapa, Pue. & $\begin{array}{l}\text { Iniciará en 2016 con } \\
\text { modelo Q5 }\end{array}$ \\
\hline
\end{tabular}

Fuente: Elaboración propia con base en datos tomados de Secretaría de Economía (2012).

Figura 2. Localización de plantas ensambladoras en México.

Vehículos Comerciales

\begin{tabular}{|c|c|c|}
\hline Empresa & Productos & Ubicación \\
\hline Scania & $\begin{array}{l}\text { Autobuses } \\
\text { foráneos, camiones, } \\
\text { tractocamiones }\end{array}$ & Tultitlán, Edo. Mex. \\
\hline Volvo GM & $\begin{array}{l}\text { Chassis autobuses } \\
\text { foráneos, camión y } \\
\text { tractocamión }\end{array}$ & Querétaro, Qro. \\
\hline Dina & Chasis y camiones & Cd. Sahagún, Hgo. \\
\hline Kenworth & $\begin{array}{l}\text { Camiones y } \\
\text { tractocamiones } \\
\end{array}$ & Mexicali, B.C. \\
\hline Internacional & $\begin{array}{l}\text { Chassis, camiones y } \\
\text { tractocamiones }\end{array}$ & Escobedo, N.L. \\
\hline Volkswagen & $\begin{array}{l}\text { Chassis y camiones } \\
\text { clase } 5\end{array}$ & Puebla, Pue. \\
\hline Giant Motors & $\begin{array}{l}\text { Camiones GF900, } \\
3600 \text { y } 6000 \\
\end{array}$ & Cd. Sahagún, Hgo. \\
\hline Man & $\begin{array}{l}\text { Autobuses foráneos, } \\
\text { camiones y } \\
\text { tractocamiones }\end{array}$ & Querétaro, Qro. \\
\hline Isuzu & $\begin{array}{l}\text { Chassis y camiones } \\
\text { clase } 4 \text { y } 6\end{array}$ & San Martín, Edo. Mex. \\
\hline $\begin{array}{l}\text { Daimler } \\
\text { Benz y Freightliner }\end{array}$ & $\begin{array}{l}\text { Chassis, autobuses } \\
\text { foráneos, camiones y } \\
\text { tractocamiones }\end{array}$ & $\begin{array}{l}\text { Santiago T., Edo. Mex. } \\
\text { García, N.L. Saltillo, } \\
\text { Coah. }\end{array}$ \\
\hline $\begin{array}{l}\text { Hino Motors } \\
\text { (Grupo Toyota) }\end{array}$ & Camiones clase 4,6 y 8 & Silao, Gto. \\
\hline
\end{tabular}

Fuente: Elaboración propia con base en datos tomados de Secretaría de Economía (2012).

\section{Autopartes}

D 1 sector de autopartes 4 se compone de las em1 presas que suministran insumos a la industria terminal armadora de automóviles principalmente, y en menor escala con autopartes de reemplazo a empresas morales y físicas que se encargan de dar mantenimiento y reparación de autos usados (Agüero, 2014).

La manufactura de autopartes en un principio estuvo dentro de la misma fabricación del automóvil, los motores e innovaciones se atribuyen a los mismos productores de autos terminados, ya que se construían de una pieza a la vez, aunque sí existía colaboración entre ellos, por ejemplo, como se mencionó en el apartado anterior, Peugeot utilizó los motores Daimler y los diseños Bugatti. Una vez iniciada la producción en serie fue necesaria la especialización en las autopartes, aunque no se dio mucho reconocimiento en esta rama, ya estaban presentes Dunlop y posteriormente Michelin en llantas (Agüero, 2014).

Inicialmente en la industria de autopartes los proveedores tenían funciones claramente delimitadas. El primer nivel, 
tier1, fabricaba componentes para otros proveedores de equipo automotriz que posteriormente ya ensamblado se entregaba de manera directa a la plantas armadoras; el segundo nivel, tier2, proveía al primer nivel de partes sencillas; $y$ el tercer nivel, tier3, surtía materias primas para equipo complementario (Cuéllar, 2012).

Las empresas de autopartes más importantes en el mundo son principalmente de Estados Unidos (31\%), Japón (28\%), Alemania (19\%) y Francia (6\%), distribuidas en el mundo (Medina, 2013).

China es el principal productor de autopartes y le siguen Japón, Estados Unidos, Alemania y México, que con una producción de 75,000 millones de dólares en 2012, desbancó a Corea del Sur, quedando como quinto productor de autopartes a nivel mundial (González, 2013).

El sector de autopartes, como parte integral de la compleja industria automotriz, presenta en México mayores oportunidades de participación de productores nacionales que el sector terminal que está compuesto de firmas extranjeras. Además de su partici- pación en la cadena productiva produciendo y suministrando al sector terminal de una gran diversidad de componentes y sistemas, también abastece al mercado de reemplazo o refacciones para automóviles usados. En México operan 89 de las 100 principales empresas a nivel mundial, de acuerdo con ProMéxico (Industria Terminal Automotriz, 2013), al contar con plantas de producción, centros de distribución y corporativos.

Este sector crece en relación directamente proporcional a la industria terminal, en 2012, de acuerdo con información de la INA, México se posicionó como el quinto productor de autopartes a nivel mundial.

A la par de la industria terminal se tejen las redes de proveeduría. Para garantizar la entrega de la producción Just in time $e^{3}$, las empresas productoras de autopartes se establecen en el entorno cercano a las plantas armadoras. En México se han instalado importantes empresas de autopartes a nivel mundial, entre las que figuran

3 En español, justo a tiempo, sistema orientado a optimizar un flujo continuo de producción integrando a las empresas proveedoras en la secuencia requerida en la cadena productiva.
Robert Bosch, Denso, Delphi, Nemak, Magna Internacional, Continental, Lear Corporation Visteon, Eaton, Valeo, Bridgestone/Firestone, Johnson Controls, Michelin, Aisin Seiki, Goodyear, Metalsa, Autolive, TRW Automotive, ThyssenKrupp, Faurecia, Siemens, San Luis Rassini, Metalsa y Brembo, entre otras, de origen norteamericano, europeo y asiático que, en los casos de los agrupamientos productivos del centro y centro-occidente de México, se ubican normalmente en los alrededores de las plantas terminales ${ }^{4}$; no así en la frontera norte de México, Ciudad Juárez-El Paso, Texas, donde son entregadas refacciones y equipo original a través de las bodegas en el lado norteamericano y de ahí a las marcas automotrices en Estados Unidos y el resto del mundo. Es importante resaltar que México es líder en la manufactura de sistemas entre los que destacan partes eléctricas, transmisiones, embragues y partes para motor.

El estudio "Competitive Alternatives KPMG's Gui-

\footnotetext{
4 Investigación de campo, Articulación Productiva del Sector Automotriz, Región Centro Occidente de México, 2013-2014.
} 
de to International Business Location 2008 Edition", de la consultora KPMG, en coordinación con Mercer, Colliers International, Economic Research Institute and Decision, coloca a México como "el país número uno para la manufactura de autopartes", y determinó en el 2011 que la manufactura de autopartes en este país ofreció un $13 \%$ de ahorro en los costos con respecto a Estados Unidos (Secretaría de Economía, 2013).

De forma análoga a la industria terminal, en su mayoría la exportación de autopartes tiene como destino Estados Unidos, que en 2011 recibió el $89 \%$ de las exportaciones de estos productos. También durante 2011 las inversiones en el sector de autopartes sumaron 996 mil millones de dólares y generaron 602,816 fuentes de empleo. De acuerdo con información de la Industria Nacional de Autopartes (Propuestas para la Agenda Automotriz 2012-2018) ${ }^{5}$, en el país existen alrededor de 600 productores de autopartes, 230 empresas

5 Propuestas para la Agenda Automotriz, Diálogo con la Industria Automotriz, documento presentado al Presidente de la República por la INA, AMDA, AMIA, AMPACT, 2013. grandes, 162 medianas y 208 pequeñas o microempresas. En 2011 registró una cifra récord de valor de la producción por 67,989 millones de dólares. En cuanto al origen del capital, la mayoría son de capital extranjero, y también encontramos nacionales y de capital mixto.

En estas empresas es común establecer divisiones entre las manufactureras de equipo original. Las de primer nivel o Tier1, hace referencia a las empresas de proveedores experimentados y confiables, que tienen capacidad de abastecer la demanda de las plantas armadoras de forma directa y tienen características de empresas de clase mundial: altos niveles de productividad, certificaciones internacionales de calidad, producción cero defectos con base en la mejora continua, alto porcentaje de automatización y utilización de la capacidad instalada de la planta, entrega justo a tiempo y capacidad de abastecer una elevada variedad de productos con reducidos inventarios y organización laboral (Villarreal-Villegas, 2004).

Por las mencionadas exigencias de los proveedores de primer nivel, estos a su vez requieren insumos de calidad y de proveedores confiables, mismos que integran el segundo nivel o Tier2. Los proveedores de primer nivel a su vez exigen de sus proveedores calidad, precio y volúmenes de entrega con seguridad para que la cadena productiva sea eficiente, para lo que se establecen acuerdos de cooperación entre estas empresas. De esta manera los proveedores Tier 1 se encargan también del diseño.

De los seiscientos productores de autopartes 450 son proveedores de equipo original, y de estos sólo el $33 \%$ son considerados como proveedores de primer nivel, es decir, tienen relación directa con las compañías de la industria terminal. El resto se compone de empresas de segundo y tercer nivel que no tienen negociación directa con las armadoras, sino que solo abastecen componentes a los proveedores de primer nivel. En lo que a especialización se refiere, la industria de autopartes presenta mayor desarrollo en la fabricación de componentes para motor, transmisión y carrocería; la experiencia es muy notoria en la fabricación de anillos, monobloques, pistones, árboles de levas, carburadores, transmi- 
siones, ejes, arneses eléctricos, rines, vidrio, estampados y partes de plástico.

Las productoras de equipo original que surten a las plantas armadoras en su mayoría forman parte de grupos multinacionales y exportan directa o indirectamente la mayor parte de su producción, además de surtir al mercado local de repuesto; incluyen áreas de investigación y desarrollo de nuevas tecnologías y productos; establecen alianzas con empresas extranjeras a fin de extender su mercado; manejan una variedad amplia de productos y cuentan con certificaciones de calidad y tiempos de entrega reducidos (Álvarez, 2002).

$\mathrm{La}$ industria automotriz es muy estricta en sus estándares de calidad, por ello la mayoría de los proveedores Tier2 y Tier3, que son empresas pequeñas y medianas que en su mayoría no cuentan con la infraestructura, personal certificado ni la tecnología para cumplir con las exigencias de calidad y volumen de las plantas armadoras de forma directa, de modo que son proveedoras de las Tier 1, las cuales necesitan la producción de varias empresas de segundo nivel para cumplir con sus compromisos de entrega; los proveedores Tier3 suministran a su vez materias primas a los Tier2.

Para hacer más eficiente este sistema se han agrupado las empresas proveedoras en parques industriales, alrededor de las plantas ensambladoras y de forma ordenada de acuerdo con el proceso productivo, o incluso dentro de la planta - si así se requiere-con un mayor control y dirección de la planta al proveedor (Rodríguez, 2013).

Un aspecto fundamental en la integración del sector ha sido la creación y, en su caso, consolidación de agrupaciones pertenecientes al sector automotriz. A continuación se mencionan algunas de ellas y su contribución a la industria a la que pertenecen.

\section{ASOCIACIONES AUTOMOTRICES}

T as empresas de la comunidad industrial automotriz en México se han agrupado en diferentes asociaciones de carácter representativo, con fines varios como formar redes de negocios y alianzas estratégicas, fuentes de información; gestiones y re- presentación ante organismos gubernamentales.

Organización Internacional de Constructores de Automóviles (OICA)

Con sede en Paris y fundada en 1919, defiende los intereses de fabricantes de vehículos, ensambladores e importadores ante organismos internacionales y en particular vinculan las asociaciones nacionales de automóviles; recopila, y difunde información entre sus miembros, instituciones y público en general (OICA, 2013).

\section{Asociación} Mexicana de la INDUSTRIA AUTOMOTRIZ (AMIA)

D una asociación civil $\checkmark$ constituida por volun$\mathcal{W}_{\text {tad }}$ propia de las empresas fabricantes de vehículos, establecida en nuestro país el 3 de enero de 1951. AMIA tiene el propósito de tener una representación exclusiva para este sector industrial prácticamente en todos los ámbitos, ante agrupaciones similares, confederaciones de cámaras así como ante los tres niveles de gobierno. 


\section{9 novaze}

Esta asociación atiende a público en general, estudiantes e investigadores dando a conocer cuestiones automotrices, evolución y estadística de los fabricantes de vehículos que la forman. AMIA está integrada por los socios fundadores Chrysler de México, Ford Motors Co., Nissan mexicana, y Volkswagen de México; como socios cooperadores Peugeot de México, Renault de México, y Suzuki Motors de México. Por último, los socios afiliados BMW de México, Honda de México y Toyota Motor Sales de México (AMIA, 2013).

Los objetivos de esta asociación (AMIA) son" Impulsar el crecimiento de la industria automotriz mexicana, manteniendo el sentido de responsabilidad a la comunidad y el medio ambiente, mediante la representación proactiva de los intereses comunes de los afiliados. Ser su portavoz, asesor y foro de concertación del cual emane y se difunda la información sectorial y el liderazgo de nuestra industria. En un marco de estricta vigilancia de la regulación aplicable y la promoción de políticas de beneficio común para los asociados. Ser una institución de excelencia y prestigio, representante de la industria automotriz mexicana, líder de opinión y conocimiento, de consulta indispensable para el desarrollo de políticas y reglamentaciones con ética y profesionalismo"

\section{INDUSTRIA NA- CiONAL de AUto- PARTES (INA) \\ sta organización fue
creada en el año de
1961 con la finalidad de} tener una representación ante el gobierno a raíz de las negociaciones del decreto de 1962 , (de integración nacional) en el que se regulaba la importación de partes automotrices a un máximo de $40 \%$ con miras a crear y fortalecer la industria de autopartes. Inicialmente se llamó Asociación Nacional de Fabricantes de Productos automotores, y, en 1981 cambió su nombre al actual Industria $\mathrm{Na}$ cional de Autopartes A.C. Tiene 950 empresas afiliadas. Su función es representar el sector de autopartes ante organismos internaciones, gobierno, cámaras sectoriales y academia (INA, 2013).

\section{Asociación MEXiCANA DE DISTRIBUIDORES DE AuTOMOTO- RES (AMDA)}

D undada en 1945 , esa asociación se conforma de más de mil seiscientos distribuidores de 42 marcas de vehículos ligeros y pesados en más de 210 ciudades en todo el país, ofreciéndoles productos y servicios para mejorar su desempeño a través de su función de apoyar a sus asociados a través de servicios integrales de calidad que faciliten su desarrollo en el sector automotriz (AMDA, 2013).

\section{REFACCIONARIAS UNIDAS DE JALIS- co A.C. (RUJAC) Dundada en 1963, esta -1 institución de carácter - local que agrupa a las} principales empresas de autopartes, tiene aproximadamente trescientos miembros. Es reconocida dentro del ámbito automotriz como una asociación de gran trayectoria, que desde 1985 respalda EXPO INTERNACIONAL RUJAC, que ha logrado convertirse en "La exposición de México" con sus 
29 emisiones anuales Ininterrumpidas (RUJAC, 2013).

Asociación NaCIONAL DE PRODUCTORES DE Autobuses, CAMIONES Y TRACTOCAMIONES (ANPACT)

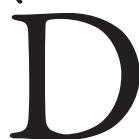
esde su fundación en 1992, representa a los fabricantes de vehículos pesados, (de 6.3 toneladas en adelante), y motores a diesel, procurando un mayor desarrollo de la industria del autotransporte en México, mediante la armonización, promoción y ejecución de estrategias para el desarrollo de esta industria (AMPACT, 2013).

\section{Cámara Na-} CIONAL DE LA INDUSTRIA DE LA TRANSFORMACIÓN (CANACINTRA)

L as cámaras y sus confederaciones representan, promueven y defienden nacional e internacionalmente las actividades de la industria y colaboran con el gobierno para lograr el crecimiento socioeconómico, así como la generación y distribución de la riqueza, (Ley de cámaras empresariales y sus confederaciones), proporcionan a sus agremiados la integración a una comunidad industrial, respaldo empresarial, facilitan redes de negocios, atención personalizada en gestión, asesoría y vinculación, alianzas estratégicas además de que es un organismo de consulta obligada. La afiliación a ésta cámara no es obligatoria, sin embargo, es un colaborador del Sistema Empresarial Mexicano, (SIEM), que administra la Secretaría de Economía y cuya inscripción si es obligatoria (CANACINTRA, 2013).

\section{Clúster Auto- MOTRIZ DE NUE- VO LEóN, A.C. (CLAUT-MTY)}

D una asociación civil 4 que integra fabricantes 1 de primer nivel de la industria automotriz e instituciones académicas y gubernamentales vinculadas a la industria automotriz. La misión es "integrar a las empresas, las universidades, los institutos tecnológicos y las organizaciones gubernamentales para colaborar en conjunto y construir una visión común para el desarrollo de la industria automotriz del estado de Nuevo León, de la que generen proyectos comunes que ayuden a incrementar la competitividad del sector y el crecimiento de nuevos negocios en la región (CLAUT, 2013).

En trabajo de investigación realizado mediante entrevistas realizadas a los directivos del CLAUT, particularmente al Director General, esta agrupación se fundó por iniciativa del ex gobernador de Nuevo León, José Natividad González Paras y por la parte empresarial el Ing. Enrique Zambrano, dueño de la empresa mexicana Metalsa, con conocido prestigio en el ramo, cuya confiabilidad le permitió integrar a más empresas importantes en el proyecto. Así se constituyó el clúster en el año 2007 con las armadoras Navistar y Daimler; las empresas Tier one: Metalsa, Nemak, Vitro, Grupo Acerero Monterrey, (que después fue adquirido por Ternium). La parte académica la integró el Tecnológico de Monterrey y la Universidad Autónoma de Nuevo León, y la parte gubernamental quedó representada por la Secretaría de Desarrollo Económico y el Instituto de 


\section{1 novare}

Innovación y Transferencia de Tecnología. Posteriormente y gracias a la influencia y peso de las empresas integrantes, se fue extendiendo la base y actualmente el clúster está formado por cuatro armadoras, Navistar, Daimler, Caterpillar y John Deere, 61 empresas Tier uno y Tier dos y quince instituciones entre académicas y de gobierno.

\section{El Punto Nacio-} NAL DE CONTACTO SECTORIAL AUTOMOTRIZ (PNCSA)

reado bajo la convoca-
toria del fondo secto-
rial de Investigación
SRE-CONACYT 2008-1, es una figura de enlace entre industria, gobierno y academia promoviendo redes de colaboración entre la comunidad científica nacional e internacional para elevar la competitividad automotriz nacional (PNCSA, 2013).

\section{LA ACADEMIA EN LA INDUSTRIA Automotriz}

T a educación superior es una condición fundamental para impulsar el desarrollo económico y social y mejorar la competitividad y consecuente prosperidad en nuestro país. En la política educativa, empezando por el artículo $3^{\circ}$ constitucional, el Estado mexicano apoyará la investigación científica y tecnológica y alentará el fortalecimiento y difusión de nuestra cultura, con el apoyo de la Ley de Ciencia y Tecnología. En cumplimiento a esta disposición, la Secretaría de Economía, (2013), menciona algunos organismos de apoyo a la innovación, como el Comité Interseccional para la innovación, facultado por la Ley de Ciencia y Tecnología para diseñar y operar la política de innovación, en el que se promueve la cooperación estrecha de gobierno, academia e industria. El Fondo de Innovación Tecnológica Apoya a las micro, pequeñas y medianas empresas, así como a personas físicas con actividad empresarial que desarrollen o adopten actividades de innovación y desarrollo tecnológico. El Consejo Nacional de Ciencia y Tecnología, (Conacyt), organismo del gobierno federal, promueve el desarrollo de la ciencia y tecnología mediante apoyos económicos para estudios de posgrado a candidatos sobresalientes, y además se encarga de elaborar las políticas de ciencia y tecnología. Por último, el Programa Nacional de Innovación, encargado de la creación y vigilancia de políticas públicas que impulsen y fortalezcan la innovación en el sector industrial y de servicios, para elevar la competitividad de la economía del país tanto en el corto, como en el mediano y largo plazos

Otros organismos involucrados en esta innovación son AERI (Alianzas Estratégicas y Redes de Innovación), este organismo es impulsado por el Conacyt, con la finalidad de apoyar la articulación de alianzas entre empresas e instituciones académicas para la formación de profesionales acorde a las necesidades de la industria; FUMEC, (Fundación México-Estados Unidos para la ciencia) y por supuesto, AMIA, (Asociación mexicana de la industria automotriz); y, por último, los Centros de Ingeniería y Diseño Automotriz, que han instalado la mayoría de las empresas ensambladoras de vehículos ligeros, y otros más creados por Conacyt, pre- 
sentes en varios estados de la República. Algunos ejemplos son: El Centro de Desarrollo Tecnológico de Nissan, Centro de Investigación, Desarrollo y Pruebas de Ingeniería Automotriz Chrysler; Centro de Investigación y Asistencia Técnica del Estado de Querétaro, (CIATEC); éste último creado con participación del gobierno federal representado por el Consejo Nacional de Ciencia y Tecnología, (Conacyt), y Laboratorios Nacionales de Fomento Industrial, (LANFI); así como participación estatal de Querétaro y por industriales del estado representados por directivos del Grupo ICA y Grupo SPICER. Actualmente en este centro se realizan proyectos para el desarrollo de la industria automotriz y de autopartes como ingeniería básica para la fabricación de maquinaria y herramentales, equipo para pruebas, sistemas de control, medición, y fabricación de prototipos de vehículos para usarse en aeropuertos (Industria de Autopartes 2012).

La educación media y superior en nuestro país está a cargo de Universidades Públicas ya sea nacional o estatales; privadas e Institutos Tecnológicos, todos estas instituciones incorporadas a la Secretaría de Educación Pública (SEP). Las instituciones educativas nacionales representativas son la Universidad Nacional Autónoma de México, el Instituto Politécnico Nacional, y la Universidad Autónoma Metropolitana, y cada estado cuenta también con universidades estatales autónomas.

El Sistema Nacional de Institutos Tecnológicos(SNIT) está constituido por 263 instituciones, de las cuales 126 son Institutos Tecnológicos federales, 131 Institutos Tecnológicos Descentralizados, cuatro Centros Regionales de Optimización y Desarrollo de Equipo (CRODE), un Centro Interdisciplinario de Investigación y Docencia en Educación Técnica (CIIDET) y un Centro Nacional de Investigación y Desarrollo Tecnológico (CENIDET). En estas instituciones, el SNIT atiende a una población escolar de 491,165 estudiantes en licenciatura y posgrado en todo el territorio nacional, incluido el Distrito Federal.

En cumplimiento a la política federal de impulso a la educación e innovación, el Programa Especial de Ciencia y Tecnología e Innovación 2013- 2018 del Conacyt, (organismo creado por el Gobierno Federal) propone triplicar la inversión en ciencia y tecnología, así como diseñar políticas públicas acordes a las necesidades de educación y a las vocaciones en cada estado; fortalecer el Sistema Nacional de Investigadores; coordinar a la Secretaría de Educación Pública y a la Secretaría de Economía en un Sistema Nacional de Ciencia, Tecnología e innovación, y, por último, integrar el sector productivo y la academia.

La industria terminal promueve la capacitación del personal dentro de la empresa introduciendo conceptos utilizados con éxito en otros países, como la manufactura esbelta cuyo objetivo es conseguir el máximo valor con recursos ajustados evitando desperdicios en todos aspectos; la mejora continua hasta la calidad total (kaizen) o 5 's, método iniciado en Toyota con la finalidad de tener espacios de trabajo más ordenados y limpios, lo que afecta favorablemente la productividad y el 
bienestar laboral. Estas técnicas son aplicadas también por los algunos proveedores Tier uno, como en el caso de las grandes manufactureras electrónicas.

En el Plan nacional de Desarrollo 2013-2018 en su apartado "Ciencia, Tecnología e Innovación" se reconoce que a pesar de la importancia de la participación económica de México en el mundo, persiste un rezago en el mercado global. La contribución del país a la producción de conocimiento no alcanza el $1 \%$ del total. Por cada mil miembros de la población económicamente activa, sólo contamos con la décima parte de investigadores que en otros países desarrollados, y tan solo 29.9 doctores graduados por cada millón de habitantes, por lo que es necesario fortalecer el capital humano.

Asimismo en el mismo Plan Nacional de Desarrollo 20122018 se plantea como un punto significativo en la enseñanza el reto de impulsar el posgrado, (que representa el nivel cumbre del sistema educativo) como una vía para formar profesionales especializados que México requiere en los dife- rentes ámbitos productivos, ya sea la industria, comercio, arte y cultura, que promuevan la investigación científica e innovación tecnológica que eleven la competitividad del país. En el apartado "México con educación de calidad en palabras de..." aporta Petra García Llamas, de Aguascalientes, Ags.: "Las universidades tecnológicas deben retomar su esencia que es la de formar técnicos superiores universitarios, pertinentes a las necesidades del sector productivo y con una amplia vinculación con éste, además de promover y difundir sus beneficios y bondades ante los sectores industriales y empresariales del país".

En cuanto a la consulta ciudadana por internet del Plan Nacional de Desarrollo, se obtuvieron las siguientes opiniones: "...aquellos que consideraron que la prioridad nacional debe ser mejorar el Sistema Educativo, revelaron que para mejorar la calidad de la educación básica en México se necesitan maestros, directores y supervisores mejor capacitados y evaluados. Esta preocupación está contenida en el espíritu de la Reforma Educativa y constituye parte integral del plan de acción de un México con Educación de Calidad. Este plan de acción también responde a la demanda de este mismo grupo de participantes, en relación con la necesidad de vincular universidades e industria, así como la importancia de alcanzar una mayor inversión en Ciencia, Tecnología e Innovación.”

\section{Metodología DE INVESTIGA- CIÓN}

ste artículo forma parte
de la investigación que
se realizó en la Región Centro Occidente de México, aporta las primeras revisiones documentales para fundamentar el trabajo de campo, se utilizó el método mixto, que integra y combina los métodos cuantitativo y cualitativo, con lo que se obtuvo una vista integral de la situación actual de la Industria Automotriz. El diseño exploratorio cualitativo incluyó una recolección de información de lo que ocurre en la realidad de las empresas dedicadas al sector Automotriz y las relaciones comerciales en su entorno. Se sustenta la base teórica de la investigación mediante consultas en 
fuentes bibliográficas, bibliotecas virtuales, textos, artículos informativos, documentos oficiales públicos, sitios web, e información estadística.

En cuanto a la investigación cuantitativa se recopiló información consistente en datos estandarizados e información estadística que permitió responder y explicar preliminarmente las preguntas de investigación. En ambos métodos, cualitativo y cuantitativo, se trabajó con un enfoque descriptivo tendiente a especificar las características y rasgos de la Industria ya señalada.

En la revisión y análisis de la información obtenida, siguiendo con el método deductivo, se inició con el universo de las particularidades de la Industria ensambladora de automóviles a nivel mundial y se centró el estudio en la Región Centro Occidente de México.

\section{CONCLUSIONES}

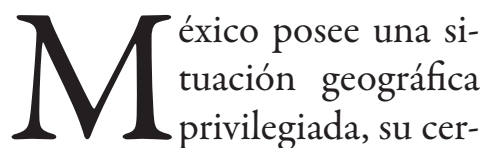
canía fronteriza con Estados Unidos de Norteamérica, los costos de operación competitivos, su experiencia probada en la fabricación de vehículos y autopartes de clase mundial, entre otros factores, hacen del país una excelente opción para atraer la inversión en este rubro.

La República Mexicana se ubica entre los diez principales productores de automóviles, camiones, partes y componentes del mundo, cuenta con una industria madura y dinámica que continúa en crecimiento. En 2014 la producción nacional rebasó los dos millones de unidades, con un crecimiento sostenido en los últimos años (AMIA, 2014). Asimismo, las exportaciones mexicanas de automóviles en los últimos años han superado las expectativas del sector nacional e internacionalmente.

Es por ello que ocho de las diez armadoras líderes de autos del mundo cuentan con plantas de ensamblaje en México, la industria automotriz se encuentra presente principalmente en las zonas Centro, Centro Occidente y Norte del País: existen nueve plantas armadoras de autos ligeros: Chrysler, Ford Motor Company, General Motors, Honda, Nissan, Toyota, Volkswagen, Mazda y Audi (en funcionamiento en 2016). En cuanto a vehículos pesados tenemos a Kenworth, Mercedes Benz, International, Cummings, Hino, Volvo, Dina, Scania e Isuzu. Además, existen más de mil empresas de autopartes establecidas en el país, la gran mayoría es de origen extranjero.

México cuenta con tres mil kilómetros de frontera con Estados Unidos, que es el mayor comprador mundial en esta industria, además de los once mil kilómetros de litoral, lo que facilita el contacto marítimo con los demás continentes, principalmente Asia y Europa.

Por otro lado el Sector Automotriz en México sigue creciendo, la interacción con empresas de diferentes tamaños y las plantas automotrices, el gobierno y la academia mantienen cierta frecuencia de relaciones lo que permite de alguna forma seguir desarrollando proyectos encaminados a lograr el enfoque hacia la innovación y tecnología, sin embargo la intensidad de las relaciones que se dan entre estos actores es en algunos casos solamente para fines comerciales y no para impulsar el dinamismo del sector tanto 
nacional como internacionalmente.

Las diferentes modalidades de articulación productiva, como clusters o redes empresariales son características de las relaciones que sostienen las plantas armadoras con sus clientes (marcas automotrices) tanto a nivel nacional e internacional, apoyados por las agrupaciones empresariales automotrices que impulsan desde su posición la intervención del estado para crear una sinergia de trabajo que además de permitirles obtener utilidades, sus beneficios se reflejen en el aumento del nivel del desarrollo humano de los trabajadores directos e indirectos y sus familias, generando empleos y propiciando con esto un mejor nivel de vida.

\section{5 novag}

\section{REFERENCIAS}

Agüero, H. (s.f.). Historia del automóvil en Asia.[es.scribd.com] Recuperado el 10 de 01 de 2014, de http://es.scribd.com/ doc/23329503/6-Historia-del-automovil-en-Asia

Álvarez, S. (2010). Top 15 productores mundiales de automóviles. [diariomotor.com] de:http://www.diariomotor. com/2010/02/17/top-15-de-productores-mundiales-de-automoviles-en-2009.

Aquino, C. (2000). Introduccion a la Economía Asiática: El desarrollo Económico del Asia oriental y lecciones para el Perú . Lima Perú: Universidad Nacional Mayor de San Marcos.

Cuéllar, J. J. (2012). El desarrollo industrial de Corea del Sur (1960-2010) Elementos explicativos y de política. Un contrapunto a la experiencia colombiana. Bogota, Colombia: Universidad Nacional de Colombia.

Álvarez, M. (2002). Cambios en la Industria Automotriz frente a la globalización. El Sector de autopartes en México. México: Universidad Nacional Autónoma de México.

Medina, S. (2013). La Industria de Autopartes. Comercio Exterior, Vol. 63.

Vidal, G y Guillén, A. (2007). Repensar la Teoría del Desarrollo en un contexto de Globalización, Glacso, enero 2007.

Vicencio, A. (2007). La Industria Automotríz en México Antecedentes, situación actual y perspectivas. México:Universidad Iberoamericana.

Villarreal, D y Villegas, M. (2005). Cambios en la localización de la industria automotriz en México 1994-2004. México: Universidad Autónoma Metropolitana de Xochimilco.

Fideicomiso para el Desarrollo De La Región Centro Occidente (FIDERCO) s.f. La región Centro Occidente en el proyecto nacional 3 de marzo 2014.

Fideicomiso para el Desarrollo de la Región Centro Occidente (FIDERCO). (s.f.). Centro Occidente, por un destino común. Recuperado el 07 de 12 de 2013, de http://centroccidente.org.mx/fiderco.html 
Gobierno del Estado de San Luis Potosí. (Marzo 2010) Plan Estatal de Desarrollo 2009-2015). San Luis Potosí, México: Organización Editorial Tangamanga.

Instituto Nacional de Estadística, Geografía e Informática, (2011) Cuéntame INEGI población de México. 05/01/2014, disponible http://cuéntame.inegi.org.mx/default.aspx\#

Instituto de Planeación del Estado de Guanajuato. Plan Estatal de Desarrollo Tomo III. (2035) Economía. 6 de febrero 2014.

ProMéxico. (2009). México, la industria automotriz.22/11/2013, folleto automotriz. Secretaría de Economía.

ProMéxico (2012). Sector Automotriz en México. Inversión y Comercio. Secretaría de Economía.

ProMéxico (2013). Industria terminal automotriz. Unidad de Inteligencia de Negocios. Secretaría de Economía. 23/12/2013.

Rodríguez, I. (2013). Proveedores 2 y 3 poco tecnificados. Manufactura. Recuperado 26/02/2013.

Secretaría de Desarrollo Económico (2013). Aguascalientes: estrategia estatal, Gobierno del Estado de Aguascalientes, México.

Secretaría de Desarrollo Económico (2012). San Luis Potosí: Industria Automotriz y de Autopartes del Estado de San Luis Potosí. 26/01/2014.

Secretaría de Economía (2013). Delegaciones y representaciones estatales, 06/02/2014. Gobierno Federal, México.

Secretaría de Gobernación (2013). Plan Nacional de Desarrollo 2013-2018. 16/10/2013. Gobierno Federal, México.

Secretaría de Planeación (2010). Plan Estatal de Desarrollo Jalisco 2030, Gobierno del Estado de Jalisco, México.

Páginas de internet

http://www.amda.mx

http://www.amia.com.mx

http://www.anpact.com.mx/quienessomos.html

http: //www.autobodymagazine.com.mx/abm_previo/2012/12/plantas-armadoras-1/Auto Body, m. (s.f.).Plantas armadoras. [autobodymagazine.com] Recuperado 02 de 11 de 2013, de: 


\section{7 novaz

http://www.autopasion18.com/HISTORIA-AUDI.htm Autopasion. (s.f.).Historia de AUDI. [autopasion18.com] de: Chrysler. (s.f.).

www.canacintra.org. $\mathrm{mx}$

http://www.claut.com.mx

http://www.chrysler.com.mx/Historia de Chrysler. [chrysler. com] Recuperado el 1 de marzo 2013.

http://www.economia.com.mx/el_milagro_mexicano.htm

Club planeta. (s.f.). El Milagro Mexicano. [Economía.com]

Recuperadol 11 de 01 de 2014

http://www.monografias.com/trabajos68/industria-automotriz/industria-automotriz.shtml\#ixzz2kFFNeD00 Cuenca, R. (23 de 12 de 2008). Industria automotriz. [monografias. com]Recuperado el 07 de 11 de 2013.

http://www.eco-finanzas.com Eco-finanzas. (s.f.). Diccionario Economía- Administración-Finanzas. [eco-finanzas.com] Recuperado el 05 de 11 de 2013.

http://eleconomista.com.mx/infografias/2013/09/06/fortaleza-industrial. El Economista. (05 de 09 de 2013). Recuperado el 22 de 01 de 2014, de Fortaleza Industrial.

http://eleconomista.com.mx/industrias/2013/04/03/mexico-quinto-productor-mundial-autopartes-inaGonzález, L. (2013) México, quinto productor mundial de autopartes. [eleconomista.com]

http://ina.com.mx

http://inventionary.blogspot.mx/2013/08/quien-invento-elautomovil.html Inventionary blogspot. (18 de agosto 2013). Desarrollo de software, emprendimientos e innovación tecnológica. [inventionaryblogspot.mx]

http://latinamericahoy.es/2012/04/17/el-sector-automotrizen-america-latina/Latin America Hoy. (2012-04-17) El sector automotriz en latinoamérica. [latinamericahoy.es] Recuperado el 18 de 12 de 2013.

http://www.mty.itesm.mx/dhcs/deptos/ri/articulos/Autosjap. htmlLópez , V. (1999) Crisis y transición de la industria automotriz japonesa. [mty.itesm.mx] Recuperado el 08 de 01 de 2014. 


\section{fua 48

http://www.pncautomotriz.org/index.php/informacion/informacion-clusters. Punto Nacional de Contacto Sectorial Automotriz. (s.f.). CLAUT.[pncautomotriz.org] de: Rodríguez, I.(15 de Enero de 2013).

http//www.manufactura.mx/industria/2013/01/15/proveedores-2-y-3-poco-tecnificados Proveedores 2 y 3 poco tecnificados. [manufactura.mx] Recuperado 06 de Enero de 2014.

http://www.manufactura.mx/automotriz/2013/12/18/jaliscobusca-reconquistar-al-sector-automotriz. Rodríguez, I. (18 de Diciembre de 2013). Jalisco busca reconquistar el sector automotriz. [manufactura.mx].

http://www.oica.net

http://www.cnnexpansion.com/negocios/2009/01/30/industria-automotriz-engorda-en-40-anos. Rozenberg, D. (31 de 01 de 2009). La industria automotriz engorda en 40 años. [cnnexpansion.com] Recuperado el 15 de 01 de 2014.

http://www.coface.com.mx.Siade P. (2013). Análisis de Riesgo, México. [coface.com].

http://www.forbes.com.mx/sites/las-diez-mejores-ciudades-para-hacer-negocios/Villafranco, G. (26 de Mayo de 2013). Las diez mejores ciudades para hacer negocios. [forbes.com].

http://www.rujac.com.mx 